\title{
Hemodynamic Characterization of Geometric Cerebral Aneurysm Templates
}

Priya Nair ${ }^{1}$, Brian W. Chong ${ }^{1,2}$, Aprinda Indahlastari ${ }^{1}$, James Lindsay ${ }^{1}$, David Dejeu ${ }^{1}$, Varsha Parthasarathy ${ }^{1}$, Justin Ryan ${ }^{1}$, Haithem Babiker ${ }^{3}$, Christopher Workman ${ }^{1}$, L. Fernando Gonzalez ${ }^{4}$, David Frakes ${ }^{1,5}$

${ }^{1}$ School of Biological and Health Systems Engineering, Arizona State University, Tempe, AZ. ${ }^{2}$ Mayo Clinic Hospital, Phoenix, AZ. ${ }^{3}$ Endovantage, Scottsdale, AZ. ${ }^{4}$ Department of Neurosurgery, Duke University School of Medicine, Durham, NC. ${ }^{5}$ School of Electrical, Computer and Energy Engineering, Arizona State University, Tempe, AZ.

Keywords: cerebral aneurysm, geometric template, hemodynamics, computational fluid dynamics, particle image velocimetry

Word Count: 4686

\section{Corresponding Author: Priya Nair}

Mailing Address: School of Biological and Health Systems Engineering, 501 E Tyler Mall, BLDG ECG RM \# 334, Arizona State University, Tempe AZ 85287

Telephone: 480-205-6530

Fax: 480-727-7624

Email: priyanair@asu.edu 


\title{
Hemodynamic Characterization of Geometric Cerebral Aneurysm Templates
}

\begin{abstract}
Hemodynamics are currently considered to a lesser degree than geometry in clinical practices for evaluating cerebral aneurysm (CA) risk and planning CA treatment. This study establishes fundamental relationships between three clinically recognized CA geometric factors and four clinically relevant hemodynamic responses. The goal of the study is to develop a more combined geometric/hemodynamic basis for informing clinical decisions. Flows within eight idealized template geometries were simulated using computational fluid dynamics and measured using particle image velocimetry under both steady and pulsatile flow conditions. The geometric factor main effects were then analyzed to quantify contributions made by the geometric factors (aneurysmal dome size (DS), dome-to-neck ratio (DNR), and parent-vessel contact angle (PV-CA)) to effects on the hemodynamic responses (aneurysmal and neck-plane root-mean-square velocity magnitude $\left(\mathrm{V}_{\mathrm{rms}}\right)$, aneurysmal wall shear stress (WSS), and cross-neck flow (CNF)). Two anatomical aneurysm models were also examined to investigate how well the idealized findings would translate to more realistic CA geometries. DNR made the greatest contributions to effects on hemodynamics including a $75.05 \%$ contribution to aneurysmal $\mathrm{V}_{\mathrm{rms}}$ and greater than $35 \%$ contributions to all responses. DS made the next greatest contributions, including a $43.94 \%$ contribution to $\mathrm{CNF}$ and greater than $20 \%$ contributions to all responses. PV-CA and several factor interactions also made contributions of greater than $10 \%$. The anatomical aneurysm models and the most similar idealized templates demonstrated consistent hemodynamic response patterns. This study demonstrates how individual geometric factors, and combinations thereof, influence CA hemodynamics. Bridging the gap between geometry and flow in this quantitative yet practical way may have potential to improve CA evaluation and treatment criteria. Agreement among results from idealized and anatomical models further supports the potential for a template-based approach to play a useful role in clinical practice.
\end{abstract}

Keywords: cerebral aneurysm, geometric template, hemodynamics, computational fluid dynamics, particle image velocimetry 
Abbreviations and Conventions: $\mathrm{CA}=$ cerebral aneurysm; DS = dome size; DNR = dome-toneck ratio; PV-CA = parent-vessel contact-angle; $\mathrm{WSS}=$ wall shear stress; $\mathrm{V}_{\mathrm{rms}}=$ root-meansquare velocity magnitude; $\mathrm{CNF}=$ cross-neck flow; $\mathrm{CFD}=$ computational fluid dynamics; PIV = particle image velocimetry; IBTA = idealized basilar tip aneurysm; ABTA = anatomical basilar tip aneurysm; SS = sum of squares; ordered pairs are used to denote results corresponding to steady and pulsatile flow conditions in the following order: (steady flow result, pulsatile flow result). 


\section{Introduction}

Aneurysmal geometry is an important factor that is considered in clinical practice during cerebral aneurysm (CA) risk evaluation and treatment planning. Extensive studies focusing on the natural history of aneurysms have identified dome size (DS) as an important predictor of growth and rupture risk. For example, CAs with DS greater than $10 \mathrm{~mm}$ in diameter have been found more prone to rupture (Ishibashi et al., 2009; Wermer et al, 2007; Brisman et al., 2006; Weibers et al, 2003). However, Forget Jr et al. reported that most ruptured aneurysms coming into the clinic had DS less than $10 \mathrm{~mm}$ (Forget Jr et al., 2001). This finding indicates that aneurysm size should not be considered as a lone predictor in evaluating CA risk. While many larger aneurysms are treated almost immediately, management of small CAs is still a gray area because the tradeoff between associated treatment and rupture risks is often unclear (especially when evaluated based on size alone) (Sonobe et al., 2010).

Aneurysmal dome-to-neck ratio (DNR) is another important geometric factor considered during treatment planning. DNR is defined as the ratio of maximum width of the aneurysmal dome to the diameter of the neck. Aneurysms with lower DNR, or wide-neck aneurysms, often present difficult treatment challenges, such as potential for coil herniation into the parent-vessel (Brinjikji et al., 2009; Weir et al., 2003). As a result, the lesions are commonly treated with assistive techniques (e.g., stent-assisted coiling or balloon remodeling). DNR also ties in with rupture risk; aneurysms with large DNR have been found more prone to rupture (Weir et al., 2003).

In addition to DS and DNR, a number of other geometric factors such as parent-vessel contact-angle (PV-CA) have also been linked to aneurysmal outcomes (Hoi et al., 2004). However, there is considerable evidence to support that hemodynamics such as aneurysmal and neck-plane root-mean-square velocity magnitude $\left(\mathrm{V}_{\mathrm{rms}}\right)$, aneurysmal wall shear stress (WSS), and cross-neck flow (CNF) may play equally or even more important roles (Babiker 
et al., 2012; Cebral et al., 2011; Mut et al., 2011; Xiang et al., 2011; Baharoglu et al., 2010; Sfroza et al., 2009; Lasheras, 2007; Cebral et al., 2005; Hoi et al., 2004, Jou et al., 2003). Not surprisingly, it is also well known that geometric factors are key determinants of CA hemodynamics (Sfroza et al., 2009). Nevertheless, unlike geometric factors, hemodynamics are still considered to a limited degree in current clinical practices for evaluating CAs. A primary reason for this lack of consideration is that hemodynamic data are often unavailable or impractical to acquire in clinical practice.

In this paper, we quantify the effects of geometric variations on aneurysmal hemodynamics in order to attribute specific hemodynamic features to geometric underliers that are routinely quantified in clinical practice. Our objective is to bridge the gap between aneurysmal anatomy and physiology by creating a link between the geometric and hemodynamic blueprints of CAs. Clinically, geometry has already been established as a primary driver of aneurysmal hemodynamics and is currently considered along with hemodynamics to stratify risk (Xiang et al., 2011; Cebral et al., 2005). However, this paper breaks new ground by quantifying the contributions that different geometric factors make to effects on hemodynamic responses. Toward this end, we employ a design of experiments based on parallel computational and bench top data. The statistical relationships between geometry and flow that emerge have potential to play a valuable role in current clinical practices for evaluating CAs and planning their treatments.

\section{Methods}

\section{Study Design}

Changes in aneurysmal geometry were parameterized in terms of the three factors (i.e., DS, DNR, and PV-CA) and hemodynamic effects were parameterized in terms of four responses (i.e., aneurysmal and neck-plane $\mathrm{V}_{\mathrm{rms}}$, aneurysmal WSS, and CNF). Basilar tip aneurysms 
(BTAs) were chosen for the study in part because aneurysms at the basilar artery bifurcation have been associated with greater risk of rupture (Loewenstein et al., 2012; Schievink, 1997). Both idealized BTA (IBTA) and anatomical BTA (ABTA) geometries were investigated.

A two-level full-factorial design was used to generate eight idealized models of CA geometries (i.e., unique combinations of the three geometric factors), as shown in Figure 1. The corners of the cube represent the different models, or geometric templates, each corresponding to high or low levels of the three factors. For example, the label $1(\mathbf{4 , 3 : 2 , 4 5 )}$ refers to the first IBTA model (IBTA-1) with $4 \mathrm{~mm} \mathrm{DS,} \mathrm{3:2} \mathrm{DNR,} \mathrm{and} \mathrm{45}{ }^{\circ} \mathrm{PV}-\mathrm{CA}$. The edges of the cube are color-coded to represent changes in a single geometric factor, with the other two factors held constant, and the arrows indicate an increase in factor value. Red edges correspond to a change in DS (increasing from $4 \mathrm{~mm}$ to $6 \mathrm{~mm}$ with the red arrow), blue edges correspond to a change in DNR (increasing from 3:2 to 2:1 with the blue arrow), and green edges correspond to a change in PV-CA (increasing from $45^{\circ}$ to $90^{\circ}$ with the green arrow).

To determine if the hemodynamic responses observed for the idealized templates could potentially be translated to more realistic patient-specific geometries, two ABTA models that were similar to two of the IBTA templates were also examined. The first ABTA model, ABTA-1, had a DS of $9.59 \mathrm{~mm}$ and DNR of 2.8 , and the second model, ABTA-2, had a DS of $14.24 \mathrm{~mm}$ and DNR of 1.4. The PV-CAs for both anatomical models were approximately $90^{\circ}$. ABTA-1 and ABTA-2 matched most closely with the geometric configurations of IBTA-4 (DS $=4 \mathrm{~mm}, \mathrm{DNR}=2$, and PV-CA $\left.=90^{\circ}\right)$ and IBTA-6 $(\mathrm{DS}=6$ $\mathrm{mm}, \mathrm{DNR}=1.5$, and PV-CA $=90^{\circ}$ ), respectively. Although the anatomical models had larger domes as compared to the corresponding idealized templates, the proportional increase in DS between ABTA-1 and ABTA-2 was comparable to the increase in DS between IBTA-4 and IBTA-6 (i.e., roughly 1.5 times). Furthermore, the DNRs of the anatomical aneurysms 
correspond, according to standard clinical convention, to narrow-neck and wide-neck aneurysms, as do IBTA-4 and IBTA-6.

\section{Computational Methods}

\section{Computational Modeling}

The eight idealized computational CA templates were designed in SolidWorks (Dassault Systèmes, Waltham, Massachusetts, USA). The aneurysms were constructed as spheres, and the inlet and outlet vessels were modeled as $4 \mathrm{~mm}$ diameter cylinders. DS, DNR, and PV-CA values were imparted to the models as reported in Figure 1 and shown in Figure 2. The directions of inflow and outflows are also represented in Figure 2 by the black arrows within the first template (IBTA-1). To create the two anatomical aneurysm models, patient-specific computed tomography image datasets were segmented and reconstructed using Mimics (Materialise, Leuven, Belgium). The underlying CT data had image matrix sizes of 512 by 512 pixels, pixel dimensions of $0.5 \mathrm{~mm}$ square, and slice thicknesses of $0.625 \mathrm{~mm}$.

\section{Computational Fluid Dynamics}

The computational models were prepared for CFD simulations using ANSYS ICEM 14.1 (ANSYS, Inc., Canonsburg, Pennsylvania, USA). The blood volume was discretized into unstructured tetrahedral elements using the Octree approach. A mesh refinement study was performed on the base model (IBTA-1) by decreasing the maximum element size from $0.5-$ $0.2 \mathrm{~mm}$ in steps of $0.05 \mathrm{~mm}$. Steady flow simulations at $3 \mathrm{~mL} / \mathrm{s}$ were performed on the different mesh sizes, and aneurysmal $\mathrm{V}_{\text {rms }}$ was calculated for each simulation. The final mesh size was deemed acceptable when the change in $\mathrm{V}_{\text {rms }}$ between subsequent mesh sizes was less than $1 \%$ and the change in WSS was less than 5\%. The final meshes (for all simulations) ranged between 6.1 and 7.1 million elements. Meshed geometries were next imported into 
ANSYS Fluent (ANSYS, Inc., Canonsburg, Pennsylvania, USA) to perform steady and pulsatile CFD simulations with inflow rates of 2, 3, and $4 \mathrm{~mL} / \mathrm{s}$, corresponding to Reynolds numbers of $247.39,371.09$, and 494.78 , respectively. These flow rates span the range of normal, exercise, and diseased flow rates reported in literature (Jou et al., 2007). The models were assumed to be rigid, and a no-slip boundary condition was imposed at the vessel wall. Blood was assumed to be incompressible and Newtonian, with a density of $1500 \mathrm{~kg} / \mathrm{m}^{3}$, and dynamic and kinematic viscosities of $3.86 \mathrm{cP}$ and $2.57 \mathrm{cSt}$, respectively. The density and viscosity were chosen to match the blood analog solution used for PIV experiments (which is described later). User-defined parabolic flow profiles were prescribed at the inlet for the steady simulations, and Ford vertebral artery flow waveforms (Ford et al., 2005) were used for the pulsatile simulations. Three cardiac cycles were simulated for the pulsatile simulations, and minimum and maximum time-step sizes of $1 \mathrm{~ms}$ and $3 \mathrm{~ms}$ were specified for the third cardiac cycle using the adaptive time-stepping technique in Fluent. Zero-pressure boundary conditions were imposed at the outlets. Pressure-velocity coupling was specified according to the SIMPLE algorithm and a second-order upwind scheme was used for momentum discretization.

\section{Experimental Methods}

A subset of the IBTA templates was chosen for validation with PIV. IBTA models 1 through 5 and 8 were selected from the initial eight computational templates using a fractional factorial design. This allowed for experimental examination of model pairs with changes in each individual geometric factor, as well as a model pair with changes in all geometric factors concurrently. Experiments were also conducted on ABTA-1 and ABTA-2. Inflow rates of 2, 3, and $4 \mathrm{~mL} / \mathrm{s}$ were explored under steady and pulsatile flow conditions (for all experimental models) in order to match the CFD simulations. 


\section{Physical Modeling}

Optically clear urethane models representing the different aneurysmal geometries for experiments were constructed using a lost-core manufacturing technique. A wax core was printed for each geometry from the corresponding computational geometry using a Solidscape R66+ 3D printer (Solidscape ${ }^{\circledR}$, Inc., Merrimack, New Hampshire, USA). The wax core was then recast in metal using a lead-tin-bismuth alloy. Clear PolyOptic 1411 urethane (Polytek Development Corp., Easton, Pennsylvania, USA) was poured around the metallic core and allowed to cure. The urethane block encapsulating the metallic core was then placed in a kiln and heated to the metal's melting point. This caused most of the core to melt away leaving a block with a hollow lumen. Remnant metal was cleared using a bath of aqua regia acid.

\section{Flow Loop}

The urethane models were mounted on a micrometer stage and connected to a flow loop for experiments. A peristaltic pump (Harvard Apparatus, Holliston, MA, USA) was used to drive the blood analog fluid, which was seeded with $8 \mu \mathrm{m}$ fluorescent microspheres (Thermo Scientific, Waltham, MA, USA) that experience peak excitation and emission at wavelengths of $542 \mathrm{~nm}$ and $618 \mathrm{~nm}$, respectively. An aqueous sodium iodide solution with a density of $1500 \mathrm{~kg} / \mathrm{m}^{3}$ and dynamic and kinematic viscosities of $3.86 \mathrm{cP}$ and $2.57 \mathrm{cSt}$, respectively, was used as the blood analog. The refractive index of the solution was matched to that of urethane $(\mathrm{n}=1.49)$. Since the pump produced a pulsatile flow pattern, an additional flow path including a variable compliance chamber was incorporated to provide steady inflow. Valves were used to switch between the two inflow paths. Equal vascular resistances were imposed 
at model outlets. Inflow and outflow rates were controlled and monitored using resistance valves and an infrared flowmeter (Omega Engineering, Inc., Stamford, CT, USA).

\section{Particle Image Velocimetry}

Volumetric flow data within the aneurysm models were acquired with a Flowmaster 3D stereo-PIV system (LaVision, Inc., Ypsilanti, Michigan, USA). A 532 nm Nd:YAG laser was used to illuminate the fluorescent microspheres flowing through the model, and two CCD cameras were used to capture particle images with a resolution of 1376 by 1060 pixels and pixel dimensions of $6.45 \mu \mathrm{m}$ square. The cameras were fitted with low-pass (frequency) filters to block laser reflections from the model. One hundred image pairs, taken at $5 \mathrm{~Hz}$, were captured at different planes within the aneurysmal volume. Images were acquired at the center plane (bisecting the aneurysmal dome, and inlet and outlet vessels) and planes displaced orthogonally from the center plane at $0.5 \mathrm{~mm}$ intervals in each direction (for a total of three planes for the smaller DS models, and five planes for the larger DS models). PIV processing applied a recursive cross-correlation algorithm within DaVis software (LaVision, Inc., Ypsilanti, Michigan, USA) where initial and final window sizes of 32 by 32 pixels and 16 by 16 pixels, respectively, were specified.

\section{Data Analysis}

\section{Computational Data Analysis}

Geometric factors were specified for the idealized models (or measured in the anatomical cases) and hemodynamic responses for each model were calculated by analyzing CFD results with Tecplot 360 (Tecplot, Inc., Bellevue, Washington, USA). WSS was averaged over the distal part of the aneurysm dome (excluding the neck) and CNF was calculated as the cumulative volume of fluid crossing the neck-plane of the aneurysm per unit time. For 
pulsatile cases, the hemodynamic responses were averaged over ten or more different time points spaced equidistant from one another throughout the third cardiac cycle. Geometric factor main effects analysis was then performed on data from the idealized templates (using JMP Pro (JMP, SAS Institute Inc., Cary, North Carolina, USA)) in order to quantify the percent contributions that individual geometric factors (and their interactions) made to effects on hemodynamic responses. Specifically, regression analysis using standard least squares estimation was used to generate the sum of squares (SS) for each of the factors and their interactions. Contributions of the geometric factors (and their interactions) toward modifying hemodynamic responses were then calculated by dividing individual SS values by the total SS. More detail on related statistical analyses can be found in Rhew et al. (Rhew and Parker, 2007). Hemodynamic responses were also compared directly between the ABTA models and their matching IBTA counterparts using Pearson correlation. Please note that in the remainder of this article ordered pairs of values are used to denote results corresponding to steady and pulsatile flow conditions in the following order: (steady flow result, pulsatile flow result).

\section{Experimental Data Analysis}

Experimental flow data were analyzed using an in-house code written in MATLAB (MathWorks, Natick, Massachusetts, USA) to calculate $\mathrm{V}_{\text {rms }}$ within the aneurysm and at the neck-plane. For pulsatile cases, these responses were averaged over three different time points of the cardiac cycle: mid systole (at $280 \mathrm{~ms}$ ), peak systole (at $570 \mathrm{~ms}$ ), and mid diastole (at $840 \mathrm{~ms}$ ). The experimental hemodynamic responses were then compared directly to the corresponding responses from CFD (for both the IBTA and ABTA models) to facilitate validation. 


\section{Results}

\section{Idealized Aneurysm Models (Templates)}

\section{Computational Results}

The changes in hemodynamic responses after changing one geometric factor across a pair of models (and holding the other two factors constant) are presented in Table 1. Increasing DS from $4 \mathrm{~mm}$ to $6 \mathrm{~mm}$ increased all hemodynamic responses (except for WSS in one model). On average over the three flow rates, increasing DS increased aneurysmal $\mathrm{V}_{\text {rms }}$ by $(74.10 \%$, $54.22 \%)$, neck-plane $\mathrm{V}_{\text {rms }}$ by $(53.76 \%, 34.93 \%)$, aneurysmal WSS by $(149.73 \%, 90.29 \%)$, and CNF by $(196.69 \%, 189.30 \%)$. In contrast, increasing DNR from 3:2 to 2:1 decreased all hemodynamic responses across all models, changing aneurysmal $\mathrm{V}_{\mathrm{rms}}$ by $(56.04 \%, 56.55 \%)$, neck-plane $\mathrm{V}_{\text {rms }}$ by $(46.53 \%, 43.16 \%)$, aneurysmal WSS by $(63.88 \%, 68.38 \%)$, and CNF by $(59.69 \%, 56.99 \%)$. Figure 3 illustrates the effects of changing DS (from black to red) and DNR (from black to green) on aneurysmal $\mathrm{V}_{\text {rms }}$ and WSS, respectively, at $3 \mathrm{~mL} / \mathrm{s}$ steady and pulsatile inflow rates. In general, increasing PV-CA from $45^{\circ}$ to $90^{\circ}$ increased only the neckplane hemodynamic responses, neck-plane $\mathrm{V}_{\mathrm{rms}}$ by $(36.98 \%, 37.75 \%)$ and CNF by $(28.71 \%$, $30.07 \%$ ). On the other hand, the $45^{\circ}$ templates generated overall greater WSS magnitudes as compared to their $90^{\circ}$ counterparts, by $(40.12 \%, 41.87 \%)$, as illustrated by the color maps in Figure 4.

Aneurysmal DNR made the greatest contributions to effects on hemodynamic responses (as much as $75.05 \%$ to pulsatile aneurysmal $\mathrm{V}_{\mathrm{rms}}$ and over $35 \%$ to every response). DS made the next greatest contributions (as much as $43.94 \%$ to pulsatile CNF and over $20 \%$ to all responses except WSS). Figure 5 illustrates the contributions that individual geometric factors and their interactions made to the effects on hemodynamic responses under steady and pulsatile inflow conditions. Although the contributions made by PV-CA in the figure were less prominent than those made by DS and DNR, PV-CA did contribute over $10 \%$ of the 
effects on aneurysmal WSS and neck-plane $\mathrm{V}_{\text {rms. }}$. Several contributions of factor interactions in the figure, PV-CA with DS or DNR for example, also exceeded 10\%.

\section{Experimental Results}

Aneurysmal and neck-plane $V_{\text {rms }}$ values were also calculated based on PIV results to validate the corresponding values calculated based on CFD results. Aneurysmal $\mathrm{V}_{\text {rms }}$ values from both PIV and CFD are shown in Figure 6 for $3 \mathrm{~mL} / \mathrm{s}$ steady and pulsatile inflows in the idealized models. Although aneurysmal $\mathrm{V}_{\text {rms }}$ values (averaged across all flow rates) were $(33.49 \%, 33.53 \%)$ greater for PIV, the general trends associated with changes in DS, DNR, and PV-CA were similar across the idealized PIV and CFD datasets. Specifically, for the six different sets of flow conditions (three flow rates and two flow types), correlations between series of simulated and experimental $\mathrm{V}_{\mathrm{rms}}$ values (from the four different geometric templates corresponding to single factor changes) ranged from 0.61 to 0.98 with an average correlation of 0.74. For example, the correlation between the steady flow PIV and CFD results presented in Figure 6 was 0.70. A qualitative comparison of the center-plane PIV and CFD flow fields from model IBTA-1 at mid-systole for a $3 \mathrm{~mL} / \mathrm{s}$ pulsatile inflow rate is presented in Figure 7.

\section{Anatomical Aneurysm Models}

\section{Computational Results}

Aneurysmal $\mathrm{V}_{\mathrm{rms}}$ and WSS values were calculated for the anatomical models (ABTA-1 and ABTA-2) based on CFD. Hemodynamic trends between ABTA-1 and ABTA-2 were similar to those between their idealized counterparts IBTA-4 and IBTA-6. Specifically, the aneurysm models with lower DS and higher DNR (i.e., ABTA-1 and IBTA-4) demonstrated lower $\mathrm{V}_{\text {rms }}$ and WSS as compared to the corresponding aneurysm models with higher DS and lower DNR (i.e., ABTA-2 and IBTA-6). The trending among anatomical and idealized counterparts 
is highlighted in Figure 8 where simulated WSS values are shown for $3 \mathrm{~mL} / \mathrm{s}$ steady and pulsatile inflows. The two data series there correlate with a value of 0.91 ; however, the average aneurysmal $\mathrm{V}_{\mathrm{rms}}$ and WSS values from the anatomical models were 2.62 and 6.54 times greater, respectively, than the corresponding values from the idealized models. Figure 9 displays streamtraces from CFD (color-coded by velocity magnitude) throughout the anatomical aneurysm geometries and their corresponding idealized templates, both at $3 \mathrm{~mL} / \mathrm{s}$ steady inflow. Features of the simulated intra-aneurysmal flow patterns were qualitatively similar between the anatomical geometries and their idealized counterparts.

\section{Experimental Results}

Aneurysmal $\mathrm{V}_{\text {rms }}$ values were also calculated for the anatomical models based on PIV in order to validate the anatomical CFD data. $\mathrm{V}_{\text {rms }}$ values were $(17.86 \%, 26.41 \%)$ greater (averaged over models and flow rates) for the experimental data as compared to the simulated data. However, $\mathrm{V}_{\text {rms }}$ increased similarly from ABTA-1 to ABTA-2 in experiments and simulations, by $(32.42 \%, 32.95 \%)$ and $(36.44 \%, 46.29 \%)$, respectively, on average across flow rates.

\section{Discussion}

Geometry is known to play an important role in CA hemodynamics and thus aneurysmal growth and/or rupture (Baharoglu et al., 2010; Lasheras, 2007; Cebral et al., 2005; Hoi et al., 2004). This study investigated the contributions made by three geometric factors (DS, DNR, and PV-CA) to effects on BTA hemodynamics. Flows within idealized geometric templates and anatomical geometries were simulated using CFD and measured using PIV. Aneurysmal WSS, $\mathrm{V}_{\text {rms }}$ within the aneurysm and at the neck, and CNF were evaluated as hemodynamic responses. To the authors' knowledge, this study represents the first time that a factorial 
design has been applied to characterize the relationships between aneurysmal geometry and hemodynamics. We believe that bridging the gap between the two bases in a quantitative yet practical way has potential to play a valuable role in clinical decision-making.

\section{Geometric Factor Main Effects on Hemodynamic Responses}

Individually, all three investigated geometric factors affected hemodynamics. Foremost among them was DNR, which is routinely evaluated in clinical practice. We observed that aneurysm templates with low DNR (3:2) generated greater hemodynamic responses (e.g., low DNR increased CNF, aneurysmal $\mathrm{V}_{\text {rms }}$, and WSS by $(59.69 \%, 56.99 \%),(56.04 \%, 56.55 \%)$, and $(63.38 \%, 68.38 \%)$, respectively, on average across flow rates). This is important because greater $\mathrm{CNF}$, aneurysmal $\mathrm{V}_{\text {rms }}$, and WSS may contribute to CA growth and/or rupture according to (Castro et al., 2009; Hoi et al., 2004).

Aneurysmal DS has been clinically established as a strong independent predictor of rupture (Weibers et al., 2003) and, like DNR, is routinely evaluated. Main effects analysis of geometric factors showed that DS made the greatest contributions to effects on CNF, and made the second greatest contributions to effects on other hemodynamic responses. More specifically, templates with larger DS had higher values of computed hemodynamic responses (e.g., high DS increased aneurysmal $\mathrm{V}_{\text {rms }}$ by $\left.(74.10 \%, 54.22 \%)\right)$. From a clinical perspective, larger aneurysms are at greater rupture risk than smaller aneurysms (Weibers et al., 2003), which correlates with our finding that higher velocities were present in largerdomed aneurysms. Not surprisingly, the combination of high DS and low DNR (i.e., large, wide-neck terminus aneurysms) produced the most extreme hemodynamic environments in this study based on all of the hemodynamic responses.

Multiple studies have reported the impact of PV-CA on hemodynamics in CAs (Baharoglu et al., 2010; Meng et al., 2007; Hoi et al., 2004). Greater inflow angles have been 
associated with elevated levels of hemodynamic responses that may promote aneurysmal growth and/or regrowth (Baharoglu et al., 2010). As expected, $\mathrm{CNF}$ and $\mathrm{V}_{\mathrm{rms}}$ at the neck were greater for the $90^{\circ}$ aneurysm templates by $(28.71 \%, 30.07 \%)$ and $(36.98 \%, 37.75 \%)$, respectively. A PV-CA of $45^{\circ}$, however, elevated aneurysmal WSS by $(40.12 \%, 41.87 \%)$, which may be due to intra-aneurysmal flow patterns like those illustrated in Figure 4 . The $45^{\circ}$ templates led to regions of flow impingement along the left (from the reader's perspective) aneurysmal wall whereas more dispersed flow patterns were observed in the $90^{\circ}$ templates. Figure 4 also shows that the combination low DS and high PV-CA produced high WSS within the aneurysm. Whether high or low WSS is detrimental remains a topic of debate (Cebral et al., 2011; Jou et al., 2008). For example, Shojima et al. reported that higher WSS plays an important role in aneurysm formation but lower WSS contributes to growth (Shojima et al., 2004).

Several combinations of geometric factors also made considerable contributions to effects on CA hemodynamics, the most prominent of which were the combinations of PV-CA with DS or DNR. The interactions of these factors made contributions of greater than $10 \%$ to effects on multiple hemodynamic responses, which shows that although individual geometric factors alone affect flow, combinations of factors may also play a role in determining hemodynamics and should thus be considered in CA treatment planning accordingly. Characterizing pre-treatment aneurysmal geometries in terms of both individual and interacting factors may provide a more complete understanding of an aneurysm's hemodynamic underpinnings for physicians to use in developing well-informed hemodynamic modification plans.

\section{Comparisons with Experimental Results}


PIV was performed on five of the eight IBTA models (and the two ABTA models) for the purpose of validation. Discrepancies were observed between $V_{\text {rms }}$ values measured with PIV and those computed with CFD for the idealized models (the PIV values were (33.49\%, $33.53 \%$ ) greater on average across flow rates). However, the trends in responses remained similar across investigated flow rates and types (the average correlation was 0.74 ) as illustrated in Figure 6. Differences between computational and experimental results may be due to differences in aneurysmal neck sizes between the computational and physical models that resulted from inexact casting of the urethane models. Based on examining a subset of the physical models, their necks were approximately $17 \%$ larger in diameter than the necks originally designed in corresponding computational models. Using the same inflow conditions for CFD and PIV would thus lead to higher intraaneurysmal velocities in the wider-neck physical models. Furthermore, PIV data were obtained from planes in the middle, higher flow region of the aneurysmal dome. CFD data, on the other hand, were computed over the entire aneurysmal domain that included lower flow regions toward the outskirts of the aneurysmal dome. The CFD results were reported for the entire aneurysmal domain in order to provide the most complete representation of aneurysmal hemodynamics. However, upon comparing the CFD and PIV data corresponding to the same fluid domains (as dictated by the span of the PIV data), the average discrepancies in aneurysmal $\mathrm{V}_{\text {rms }}$ were $(22.87 \%$, $25.18 \%$ ). We expect that these smaller magnitude discrepancies, which are also on the order of those observed for the anatomical cases, i.e., $(17.86 \%, 26.41 \%)$, resulted from the aforementioned differences between the physical and computational geometries.

\section{Comparisons among Idealized and Anatomical Findings}

To understand how the findings from idealized templates could potentially be translated to a more clinically relevant scenario, the two ABTA models were investigated. The simulated 
intra-aneurysmal flow patterns, represented in Figure 9, were qualitatively similar between the anatomical geometries and their idealized counterparts. There were magnitude differences between the anatomical and idealized hemodynamic responses (aneurysmal $\mathrm{V}_{\mathrm{rms}}$ and WSS values were 1.39 and 2.83 times greater on average in the anatomical cases), but such differences were expected given the dome size differences between the anatomical and idealized model sets. Further, as quantified earlier and illustrated in Figure 8, hemodynamic changes observed between the ABTA models and between their idealized counterparts were consistent. Specifically, simulated aneurysmal $\mathrm{V}_{\text {rms }}$ and WSS values varied in the same directions between ABTA-1 and ABTA-2 and between IBTA-4 and IBTA-6, without exception, regardless of flow rate or type. This similarity in findings from the idealized and the anatomical aneurysm models indicates that characterizing the hemodynamic effects of aneurysmal geometry using templates has potential for utility in CA evaluation. Comparable approaches have demonstrated success in applying template-based methods to characterize hemodynamics in even more complex cardiovascular geometries such as the Fontan connection (Pekkan et al., 2009).

\section{Limitations and Future Work}

This study is subject to several limitations. First, a rigid wall assumption was used in the simulations and experiments. While using compliant models could be beneficial, previous studies have shown that the main factor driving aneurysmal hemodynamics is vessel geometry and that vessel compliance is secondary (or even tertiary) (Cebral et al., 2011; Zhao et al., 2000). Second, the fluid mesh did not include near-wall refinement. Although nearwall mesh refinement would improve simulated WSS accuracy, given the very large WSS changes among different cases as reported in Table 1 (i.e., up to $473 \%$ and $(91.42 \%, 72.67 \%)$ in magnitude on average), it is very unlikely that including near-wall refinement would affect 
any of the conclusions of this study. Further, WSS was considered in the mesh refinement study. Other assumptions of the study included Newtonian blood flow and equal zeropressure boundary conditions at the outlets. Although the outlet boundary conditions used in simulations were not physiologic in an absolute sense, assuming equal pressures at the outlets is reasonable given the typically similar distal resistances in the cerebrovascular circulation (Rayz et al., 2014). To ensure that simplifying the absolute pressure values did not affect flow splits at the outlets considerably, an additional BTA simulation was run at more physiologic outlet pressure values, and the resulting flow splits varied from the simplified case by less than $1 \%$. It is well known that more advanced outlet boundary conditions such as a three-element Windkessel (RCR) model can improve simulation accuracy, and do become more important when only small portions of the cerebrovascular system are included in the simulated fluid domain (as in this study). However, such boundary conditions are most important for deformable wall simulations and in cardiovascular regions where, unlike the cerebrovasculature, compliance is highly prevalent (Marsden and Esmaily-Moghadam, 2015).

Lastly, it is also noteworthy that the results of this study apply only to specific geometric CA templates. One of the anatomical CAs from our database included an aneurysm that was oriented normal to the plane formed by the parent and outlet vessels at the bifurcation (rather than within that plane, on top of the bifurcation). As a result, the hemodynamics in that CA (which were investigated in a different study) did not match closely with any of the idealized templates. Future work will include performing different endovascular treatments on geometric templates to investigate the combined effects of geometry and treatment on hemodynamic outcomes, as well as incorporating more realistic boundary conditions. Alternative approaches to elucidating the hemodynamic effects of geometric factors, including optimization-based approaches, may also be pursued. 


\section{Conclusions}

We quantified the effects of three geometric factors on hemodynamic responses in CAs using CFD simulations. The CFD results were also validated against data from PIV experiments. Geometric factor main effects analysis showed that DNR had the greatest impact on hemodynamics, followed by DS. While the impact of PV-CA was lesser, its effects were greatest on WSS among the hemodynamic responses examined. Several factor interactions, e.g., PV-CA with DS or DNR, also made notable contributions to hemodynamic responses. Overall, the combination of large DS and small DNR (i.e., large, wide-neck terminus aneurysms) produced the most extreme hemodynamic environments encountered in the study. While some of these findings are intuitive, confirming them through a 2-level, fullfactorial experimental design provides an objective basis that complements clinical experience. Bridging the gap between geometry and flow in this quantitative yet practical way may therefore have potential to improve CA evaluation and treatment criteria. The agreeable results from investigating two anatomical CA geometries further support the potential for our template-based approach to play a useful role in the clinic.

\section{Acknowledgements}

The authors acknowledge funding from the American Heart Association Beginning Grant in Aid (\#11BGIA7970009), the National Science Foundation CAREER Award (\#1151232), the Brain Aneurysm Foundation Research Grant, and the Women in Philanthropy Society Category B Grant. 


\section{References}

Babiker, M.H., Gonzalez, L.F., Ryan, J., Albuquerque, F., Collins, D., Elvikis, A., Frakes, D.H. 2012. Influence of stent configuration on cerebral aneurysm fluid dynamics. Journal of biomechanics 45(3), 440-447.

Baharoglu, M.I., Schirmer C.M., Hoit D.A., Gao, B.L., Malek, A.M., 2010. Aneurysm inflow-angle as a discriminant for rupture in sidewall cerebral aneurysms morphometric and computational fluid dynamic analysis. Stroke 41(7), 1423-1430.

Brinjikji, W., Cloft, H.J., Kallmes, D.F., 2009. Difficult aneurysms for endovascular treatment: overwide or undertall?. American Journal of Neuroradiology 30(8), 15131517.

Brisman, J.L., Song, J.K., Newell, D.W., 2006. Cerebral aneurysms. New England Journal of Medicine 355(9), 928-939.

Castro, M., Putman, C., Radaelli, A., Frangi, A., Cebral, J., 2009. Hemodynamics and rupture of terminal cerebral aneurysms. Academic Radiology 16(10), 1201-1207.

Cebral, J.R., Castro, M.A., Burgess, J.E., Pergolizzi, R.S., Sheridan, M.J., Putman, C.M., 2005. Characterization of cerebral aneurysms for assessing risk of rupture by using patient-specific computational hemodynamics models. American Journal of Neuroradiology 26(10), 2550-2559.

Cebral, J.R., Mut, F., Weir, J., Putman, C.M., 2011. Association of hemodynamic characteristics and cerebral aneurysm rupture. American Journal of Neuroradiology 32(2), 264-270.

Ford, M.D., Alperin, N., Lee, S.H., Holdsworth, D.W., Steinman, D.A., 2005. Characterization of volumetric flow rate waveforms in the normal internal carotid and vertebral arteries. Physiological Measurement 26(4), 477. 
Forget Jr, T.R., Benitez, R., Veznedaroglu, E., Sharan, A., Mitchell, W., Silva, M., Rosenwasser, R.H., 2001. A review of size and location of ruptured intracranial aneurysms. Neurosurgery 49(6), 1322-1326.

Hoi, Y., Meng, H., Woodward, S.H., Bendok, B.R., Hanel, R.A., Guterman, L.R., Hopkins, L.N., 2004. Effects of arterial geometry on aneurysm growth: three-dimensional computational fluid dynamics study. Journal of Neurosurgery 101(4), 676-681.

Ishibashi, T., Murayama, Y., Urashima, M., Saguchi, T., Ebara, M., Arakawa, H., Irie, K., Takao, H., Abe, T., 2009. Unruptured intracranial aneurysms incidence of rupture and risk factors. Stroke 40(1), 313-316.

Jou, L.D., Lee, D.H., Morsi, H., Mawad, M.E., 2008. Wall shear stress on ruptured and unruptured intracranial aneurysms at the internal carotid artery. American Journal of Neuroradiology 29(9), 1761-1767.

Jou, L.D., Mohamed, A., Lee, D.H., Mawad, M.E., 2007. 3D rotational digital subtraction angiography may underestimate intracranial aneurysms: findings from two basilar aneurysms. American Journal of Neuroradiology 28(9), 1690-1692.

Jou, L.D., Quick, C.M., Young, W.L., Lawton, M.T., Higashida, R., Martin,A., Saloner, D. 2003. Computational approach to quantifying hemodynamic forces in giant cerebral aneurysms. American Journal of Neuroradiology 24(9), 1804-1810.

Lasheras, J.C., 2007. The biomechanics of arterial aneurysms. Annual Review of Fluid Mechanics 39, 293-319.

Loewenstein, J.E., Gayle, S.C., Duffis, E.J., Prestigiacomo, C.J., Gandhi, C.D., 2012. The natural history and treatment options for unruptured intracranial aneurysms. International Journal of Vascular Medicine 2012.

Marsden, A.L., Esmaily-Moghadam, M. 2015. Multiscale Modeling of Cardiovascular Flows for Clinical Decision Support. Applied Mechanics Reviews 67(3), 030804. 
Meng, H., Wang, Z., Hoi, Y., Gao, L., Metaxa, E., Swartz, D.D., Kolega, J., 2007. Complex hemodynamics at the apex of an arterial bifurcation induces vascular remodeling resembling cerebral aneurysm initiation. Stroke 38(6), 1924-1931.

Mut, F., Löhner, R., Chien, A., Tateshima, S., Viñuela, F., Putman, C., Cebral, J.R. 2011. Computational hemodynamics framework for the analysis of cerebral aneurysms. International journal for numerical methods in biomedical engineering 27(6), 822-839.

Pekkan, K., Dasi, L.P., de Zélicourt, D., Sundareswaran, K.S, Fogel, M.A., Kanter, K.R., Yoganathan, A.P., 2009. Hemodynamic performance of stage-2 univentricular reconstruction: Glenn vs. hemi-Fontan templates. Annals of Biomedical Engineering 37(1), 50-63.

Rayz, V.L., Abla, A., Boussel, L., Leach, J.R., Acevedo-Bolton, G., Saloner, D., Lawton, M. T., 2014. Computational Modeling of Flow-Altering Surgeries in Basilar Aneurysms. Annals of Biomedical Engineering 1-13.

Rhew, R.D., Parker, P.A., 2007. A Parametric Geometry Computational Fluid Dynamics (CFD) Study Utilizing Design of Experiments (DOE). In 2007 US Air Force T\&E Days.

Schievink, W.I., 1997. Intracranial aneurysms. New England Journal of Medicine 336(1), 2840.

Sforza, D.M., Putman, C.M., Cebral, J.R., 2009. Hemodynamics of cerebral aneurysms. Annual Review of Fluid Mechanics 41, 91.

Shojima, M., Oshima, M., Takagi, K., Torii, R., Hayakawa, M., Katada, K., Morita, A., Kirino, T., 2004. Magnitude and role of wall shear stress on cerebral aneurysm computational fluid dynamic study of 20 middle cerebral artery aneurysms. Stroke $35(11), 2500-2505$. 
Sonobe, M., Yamazaki, T., Yonekura, M., Kikuchi, H., 2010. Small Unruptured Intracranial Aneurysm Verification Study SUAVe Study, Japan. Stroke 41(9), 1969-1977.

Weir, B., Amidei, C., Kongable, G., Findlay, J.M., Kassell, N.F., Kelly, J., Dai, L., Karrison, T.G., 2003. The aspect ratio (dome/neck) of ruptured and unruptured aneurysms. Journal of Neurosurgery 99(3), 447-451.

Wermer, M.J., van der Schaaf, I.C., Algra, A., Rinkel, G.J., 2007. Risk of Rupture of Unruptured Intracranial Aneurysms in Relation to Patient and Aneurysm Characteristics An Updated Meta-Analysis. Stroke 38(4), 1404-1410.

Wiebers, D. O., International Study of Unruptured Intracranial Aneurysms Investigators., 2003. Unruptured intracranial aneurysms: natural history, clinical outcome, and risks of surgical and endovascular treatment. The Lancet 362(9378), 103-110.

Xiang, J., Natarajan, S.K., Tremmel, M., Ma, D., Mocco, J., Hopkins, L.N., Siddiqui, A.H., Levy, E.I., Meng, H., 2011. Hemodynamic-morphologic discriminants for intracranial aneurysm rupture. Stroke 42(1), 144-152.

Zhao, S. Z., Xu, X.Y., Hughes, A.D., Thom, S.A., Stanton, A.V., Ariff, B., Long, Q., 2000. Blood flow and vessel mechanics in a physiologically realistic model of a human carotid arterial bifurcation. Journal of biomechanics 33(8), 975-984. 


\section{List of Tables}

1. Percentage changes in hemodynamic responses after changing one geometric factor, keeping the other two factors constant, for all different flow rates

\section{List of Figures}

1. Cube representing a two-level full-factorial experimental design based on three geometric factors: aneurysmal dome size, dome-to-neck ratio, and parent-vessel contact-angle

2. Computational idealized basilar tip aneurysm models corresponding to the corners of the cube in Figure 1. The numbers in red, green, and blue represent the dome size (in $\mathrm{mm}$ ), dome-to-neck ratio, and parent-vessel contact-angle (in degrees), respectively. The black arrows in IBTA-1 indicate the directions of inflow and outflows.

3. (a) Effects of dome size change on aneurysmal $V_{\text {rms }}$ (keeping dome-to-neck ratio and parent-vessel contact-angle constant within grouped bars), (b) effects of dome-to-neck ratio change on aneurysmal wall shear stress (keeping dome size and parent-vessel contact-angle constant within grouped bars). Solid and patterned colors correspond to $3 \mathrm{~mL} / \mathrm{s}$ steady and pulsatile CFD simulations, respectively, in the idealized model templates.

4. Illustrations of intraaneurysmal flow patterns from CFD with black streamtraces and color-coded wall shear stress overlays at $3 \mathrm{~mL} / \mathrm{s}$ steady inflow

5. Contributions made by individual geometric factors, and their interactions, to effects on simulated hemodynamic responses under steady (solid boxes) and pulsatile (patterned boxes) flow conditions in the idealized model templates: (a) aneurysmal wall shear stress, (b) aneurysmal $\mathrm{V}_{\text {rms }}$, (c) neck-plane $\mathrm{V}_{\text {rms }}$, and (d) cross neck flow

6. Comparisons between aneurysmal $\mathrm{V}_{\mathrm{rms}}$ values from PIV and CFD when changing dome size (black to red), dome-to-neck ratio (back to green), and parent-vessel 
contact-angle (black to blue). Solid and patterned colors correspond to $3 \mathrm{~mL} / \mathrm{s}$ steady and pulsatile inflows, respectively, in the idealized model templates.

7. (a) PIV and (b) CFD flow fields color-coded by velocity magnitude in model IBTA-1 at mid-systole for a $3 \mathrm{~mL} / \mathrm{s}$ pulsatile inflow

8. Simulated aneurysmal wall shear stress in anatomical and idealized geometries at 3 $\mathrm{mL} / \mathrm{s}$ steady and pulsatile inflows

9. Illustrations of intraaneurysmal flow patterns from CFD with streamtraces colorcoded by velocity magnitude. Results correspond to (top) anatomical aneurysm geometries and (bottom) idealized templates, both at $3 \mathrm{~mL} / \mathrm{s}$ steady inflow. 
Figure 1

$4(4,2: 1,90) \quad 8(6,2: 1,90)$

$3(4,2: 1,45)$

$7(6,2: 1,45)$

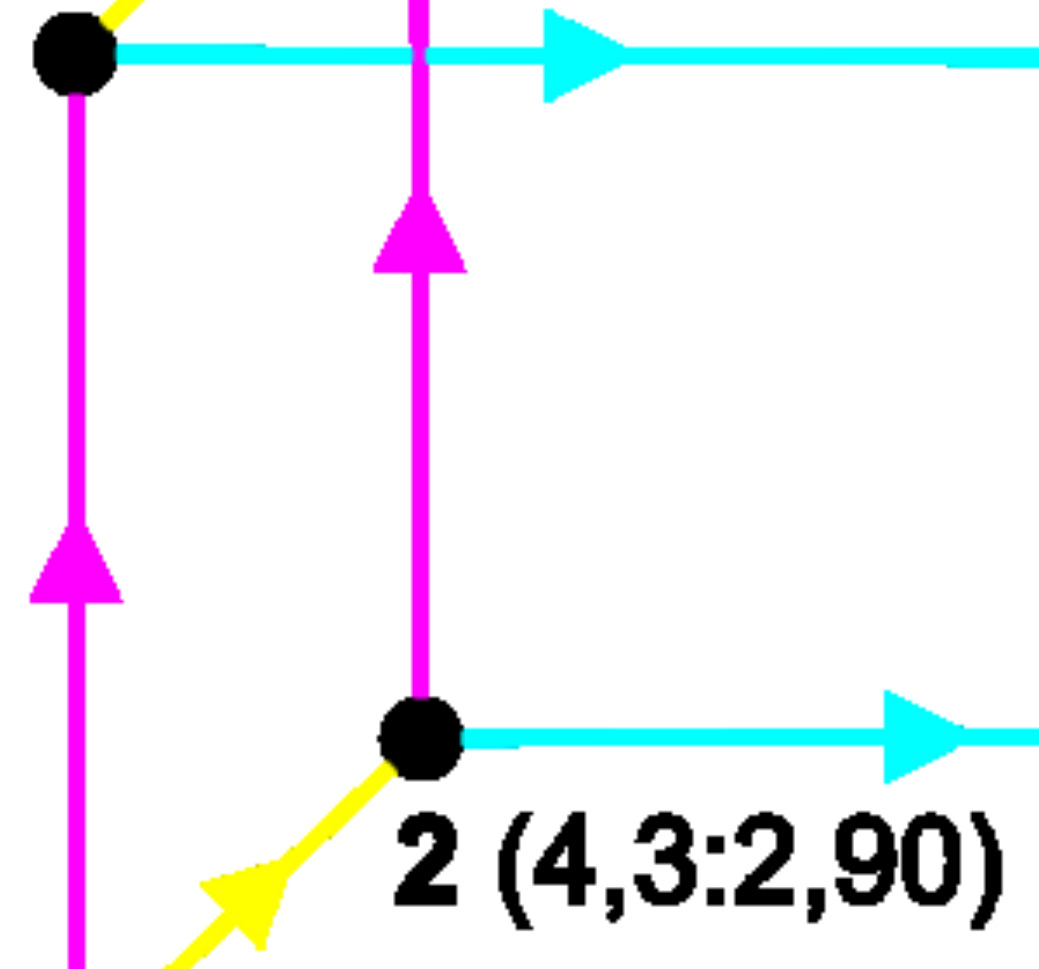

$6(6,3: 2,90)$

$1(4,3: 2,45)$

$5(6,3: 2,45)$ 


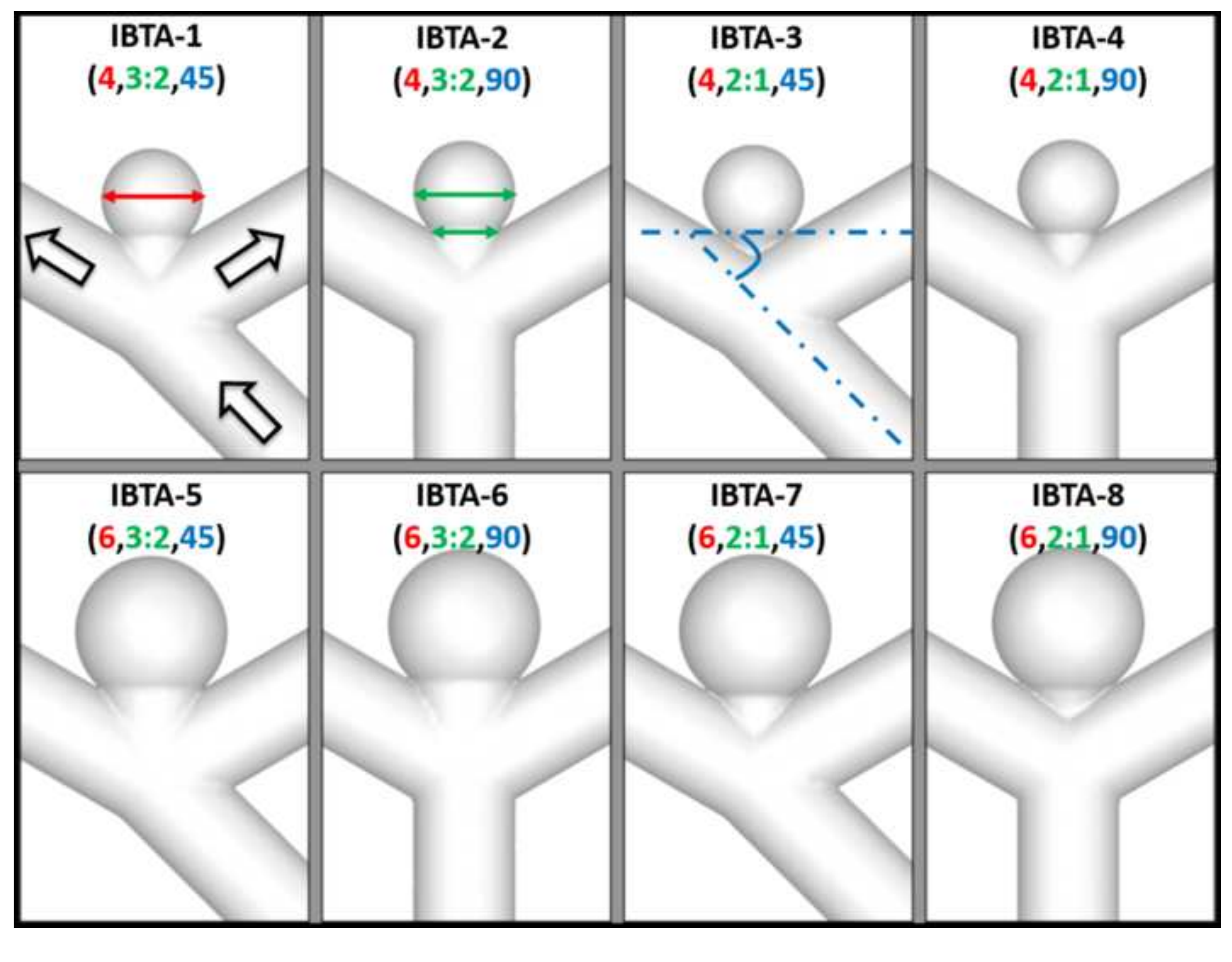

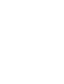

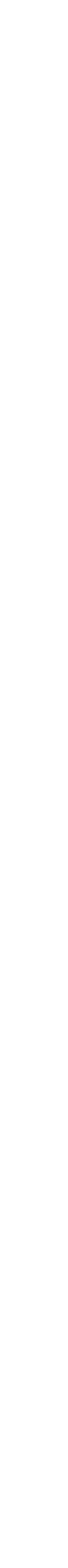

(

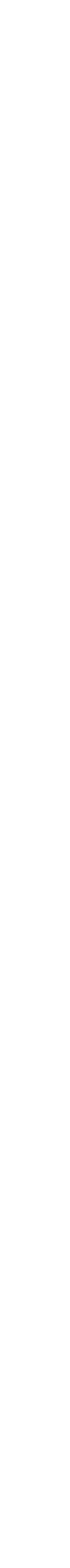




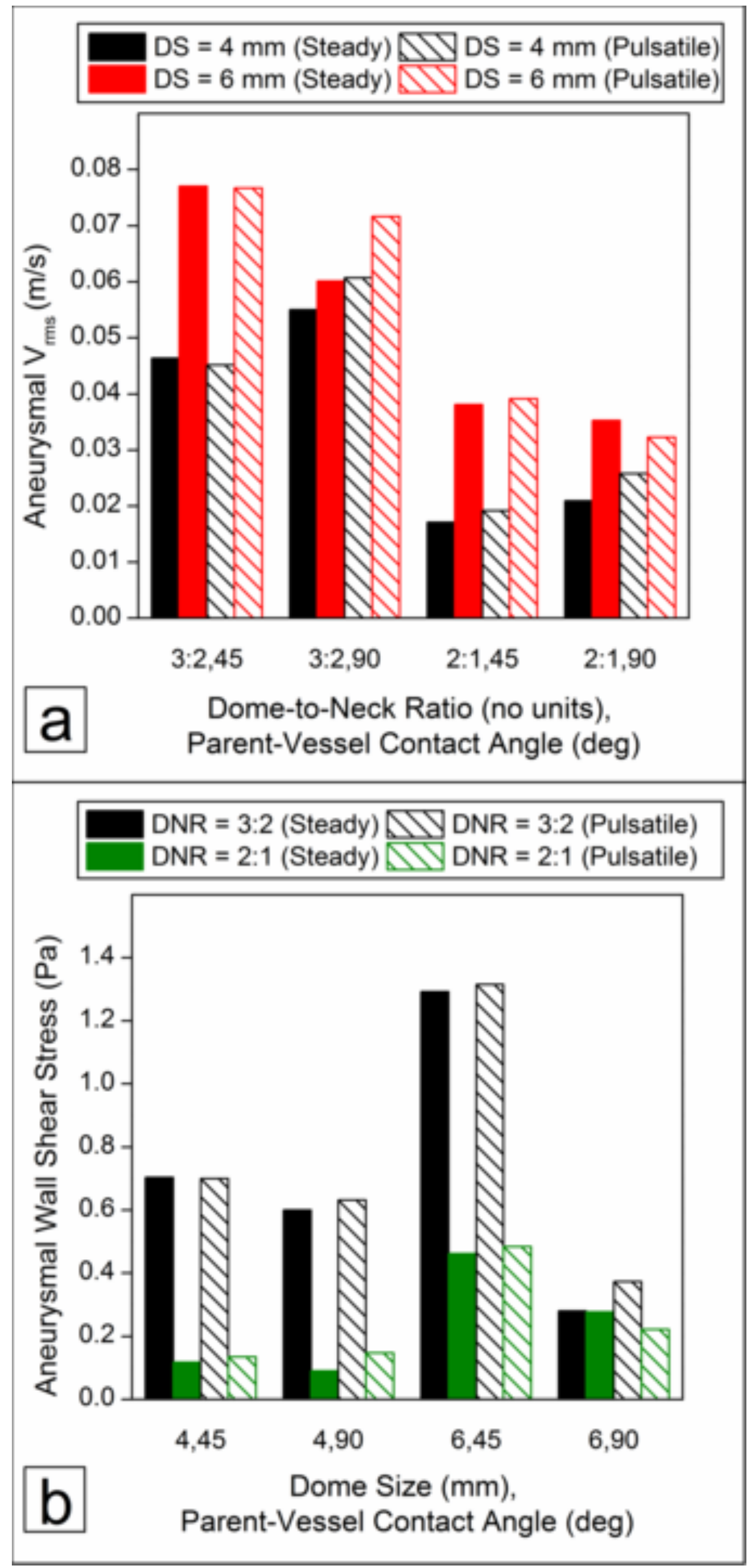



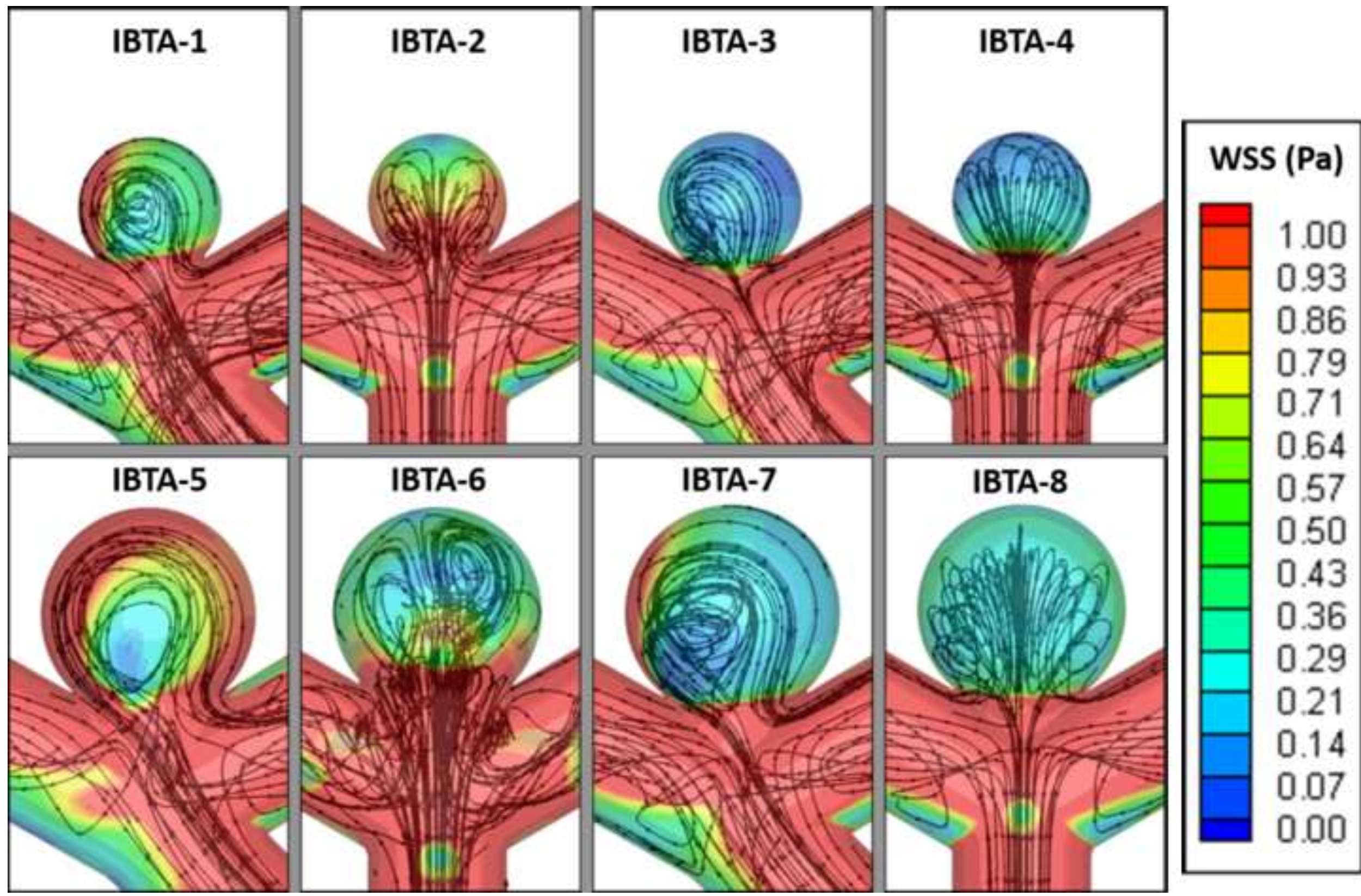
Figure 5

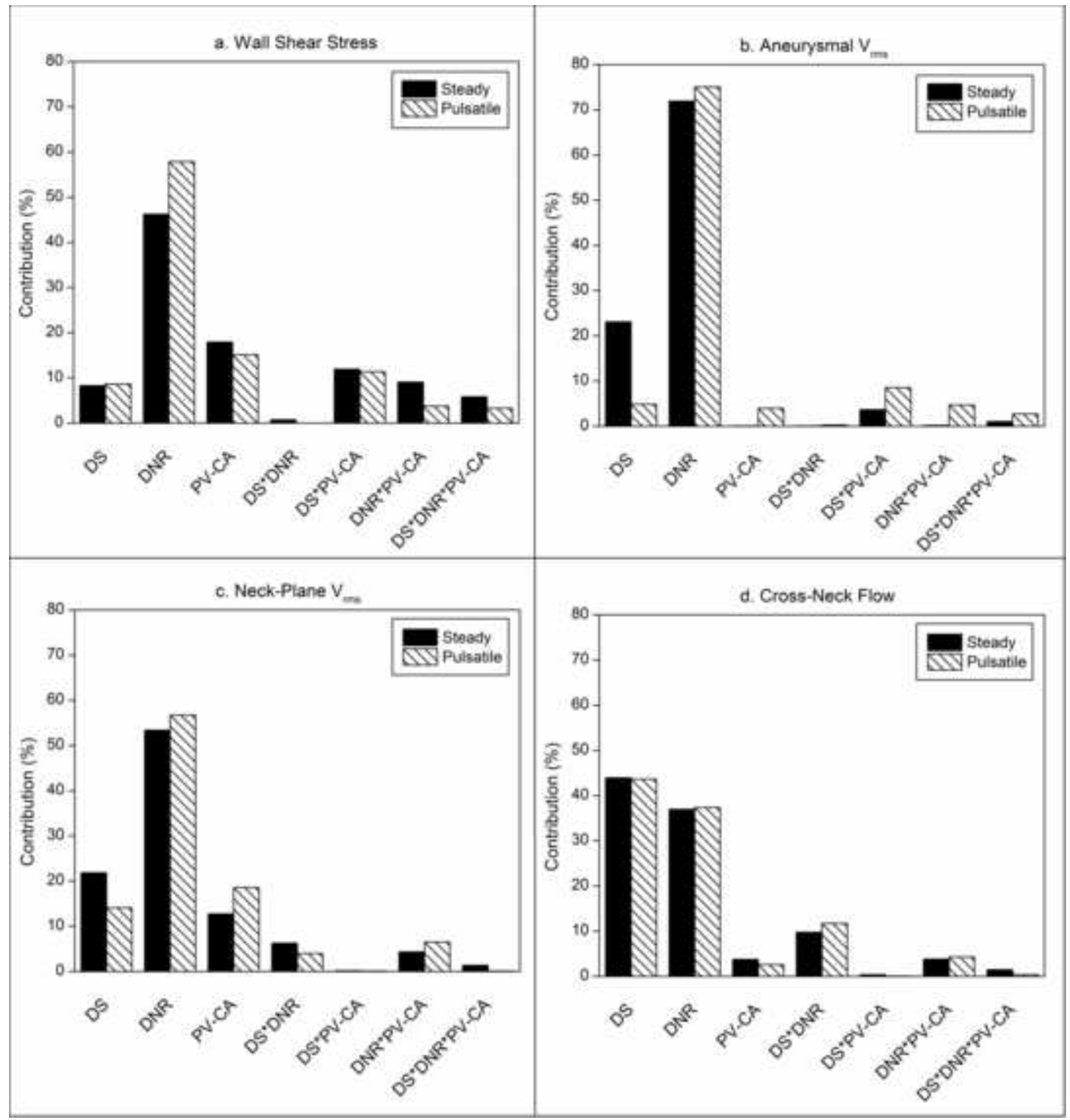




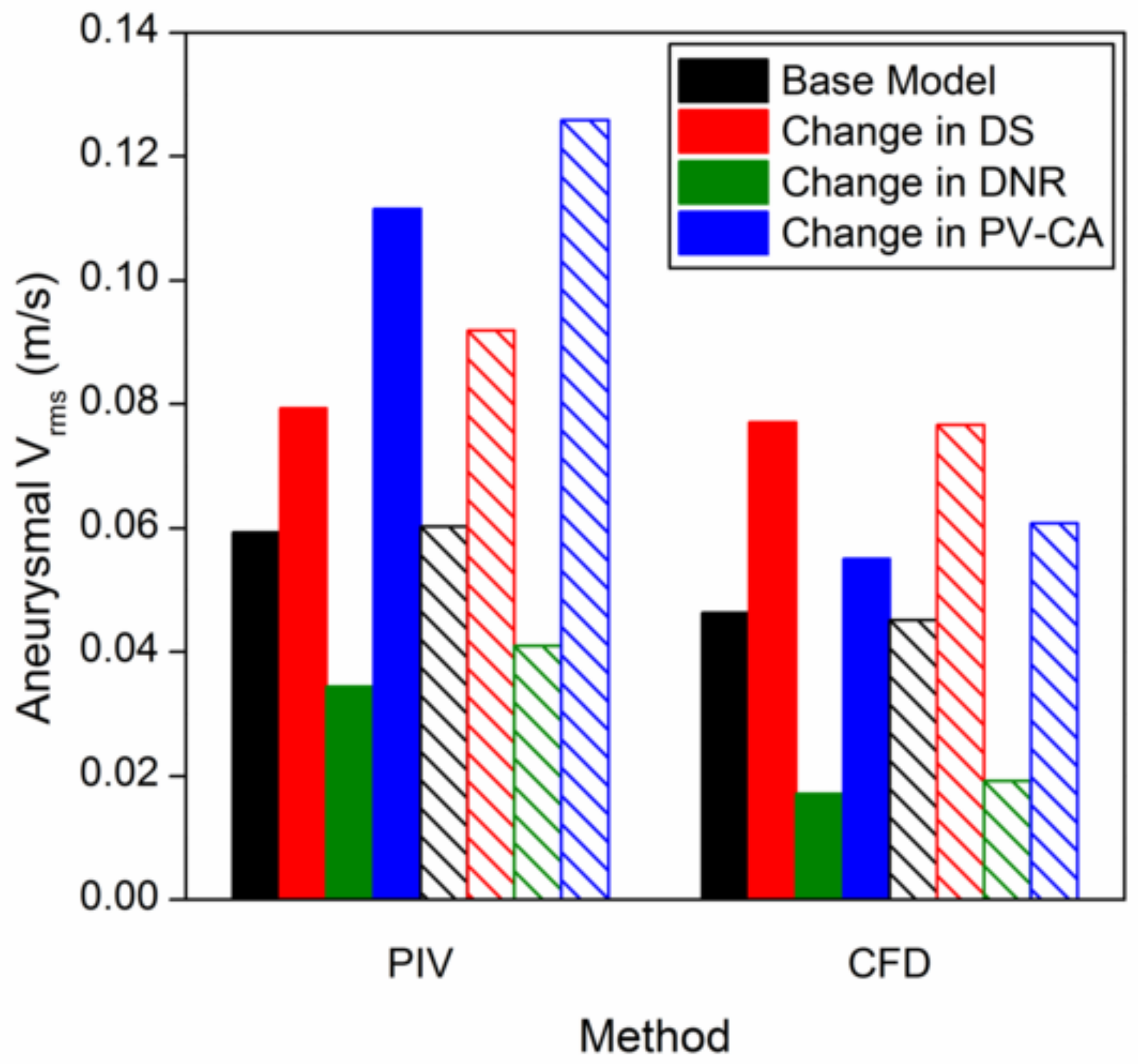




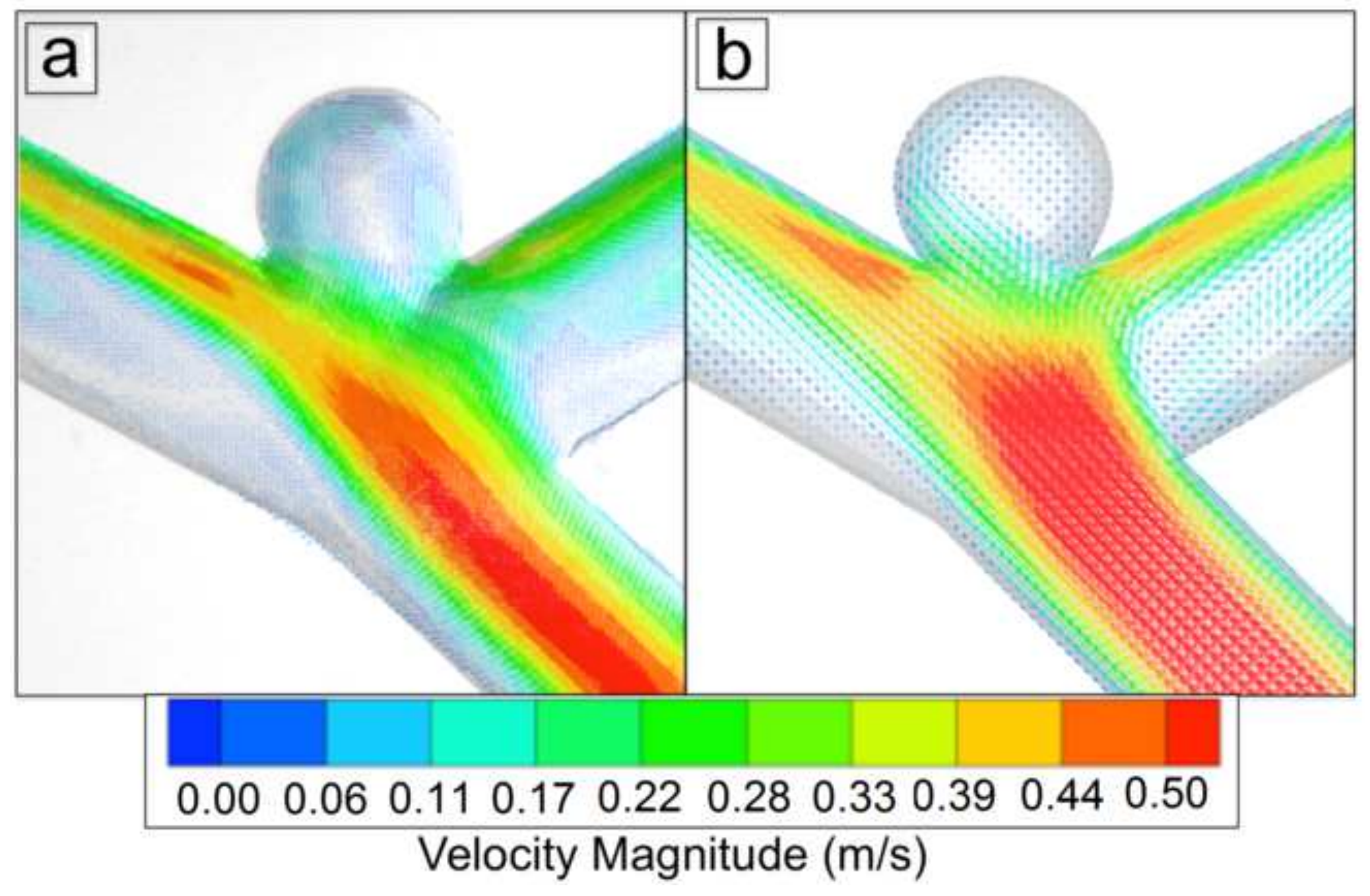

\section{Velocity Magnitude (m/s)}




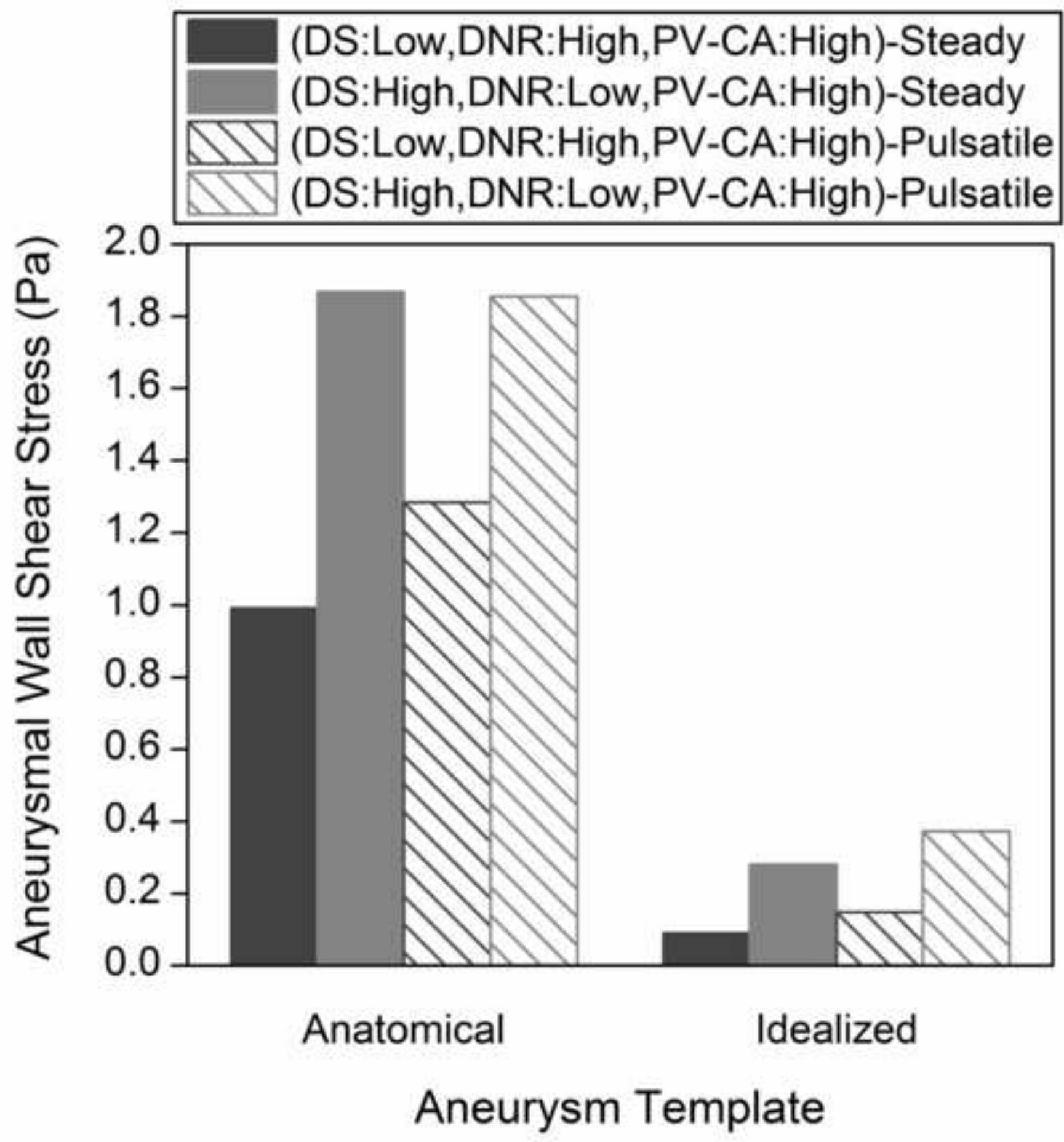




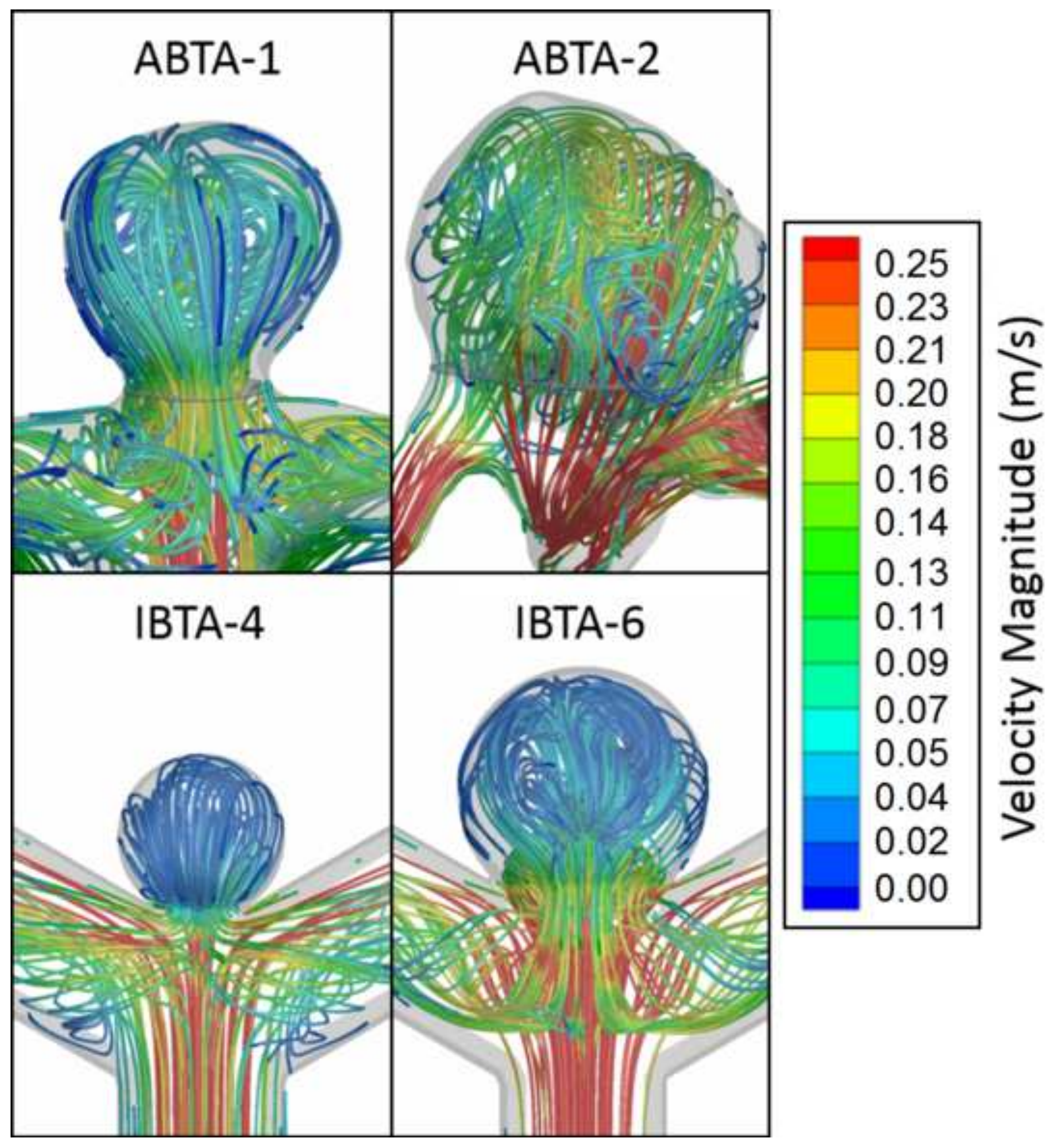




\begin{tabular}{|c|c|c|c|c|c|c|c|c|c|c|}
\hline \multirow[t]{2}{*}{$\begin{array}{l}\text { Changed } \\
\text { Factors }\end{array}$} & \multirow{2}{*}{$\begin{array}{c}\text { Constant } \\
\text { Factors } \\
\text { (Factor1; } \\
\text { Factor2) }\end{array}$} & \multirow{2}{*}{$\begin{array}{c}\text { Flow } \\
\text { Rate } \\
(\mathrm{mL} / \mathrm{s})\end{array}$} & \multicolumn{2}{|c|}{$\begin{array}{c}\text { Aneurysmal WSS } \\
(\%)\end{array}$} & \multicolumn{2}{|c|}{$\begin{array}{c}\text { Aneurysmal } \mathbf{V}_{\text {rms }} \\
(\%)\end{array}$} & \multicolumn{2}{|c|}{$\begin{array}{c}\text { Neck-PlaneV } \\
(\%)\end{array}$} & \multicolumn{2}{|c|}{$\begin{array}{l}\text { CNF } \\
(\%)\end{array}$} \\
\hline & & & Steady & Pulsatile & Steady & Pulsatile & Steady & Pulsatile & Steady & Pulsatile \\
\hline \multirow{12}{*}{$\begin{array}{l}\text { Dome } \\
\text { Size } \\
4 \mathrm{~mm} \\
\text { to } \\
6 \mathrm{~mm}\end{array}$} & \multirow{3}{*}{$\left(3: 2 ; 45^{\circ}\right)$} & 2 & 137.70 & 125.95 & 94.93 & 92.69 & 88.07 & 70.51 & 241.55 & 220.29 \\
\hline & & 3 & 83.74 & 87.94 & 66.31 & 69.89 & 68.52 & 57.59 & 204.64 & 265.18 \\
\hline & & 4 & 64.22 & 65.93 & 54.22 & 54.88 & 60.43 & 47.70 & 189.34 & 376.88 \\
\hline & \multirow{3}{*}{$\left(3: 2 ; 90^{\circ}\right)$} & 2 & -30.91 & -37.18 & 38.36 & 51.52 & 69.32 & 30.45 & 74.59 & 174.38 \\
\hline & & 3 & -53.49 & -40.98 & 9.36 & 17.95 & 78.37 & 36.21 & 267.69 & 149.57 \\
\hline & & 4 & -41.79 & -15.18 & 10.20 & -35.72 & 35.33 & 31.46 & 196.34 & 160.19 \\
\hline & \multirow{3}{*}{$\left(2: 1 ; 45^{\circ}\right)$} & 2 & 473.34 & 144.00 & 168.72 & 124.36 & 86.08 & 40.00 & 293.87 & 216.26 \\
\hline & & 3 & 296.48 & 259.37 & 122.71 & 104.00 & 68.92 & 34.62 & 250.00 & 204.97 \\
\hline & & 4 & 224.45 & 190.60 & 102.44 & 85.25 & 62.05 & 29.83 & 233.24 & 177.80 \\
\hline & \multirow{3}{*}{$\left(2: 1 ; 90^{\circ}\right)$} & 2 & 289.18 & 164.90 & 98.28 & 33.48 & 16.02 & 23.90 & 154.22 & 149.31 \\
\hline & & 3 & 210.01 & 51.21 & 68.60 & 25.40 & 6.98 & 1.50 & 130.27 & 92.86 \\
\hline & & 4 & 143.85 & 86.94 & 55.12 & 26.93 & 4.97 & 15.43 & 124.50 & 83.89 \\
\hline \multirow{12}{*}{$\begin{array}{l}\text { Dome } \\
\text {-to- } \\
\text { Neck } \\
\text { Ratio }\end{array}$} & \multirow{3}{*}{$\left(3: 2 ; 45^{\circ}\right)$} & 2 & -88.18 & -72.54 & -68.23 & -61.05 & -45.54 & -33.54 & -60.45 & -54.86 \\
\hline & & 3 & -83.44 & -80.76 & -63.03 & -57.52 & -41.84 & -31.17 & -57.40 & -49.43 \\
\hline & & 4 & -80.02 & -76.78 & -60.03 & -54.80 & -40.45 & -30.73 & -56.38 & -10.74 \\
\hline & \multirow{3}{*}{$\left(3: 2 ; 90^{\circ}\right)$} & 2 & -91.19 & -88.94 & -69.21 & -49.88 & -44.73 & -49.96 & -65.86 & -69.37 \\
\hline & & 3 & -85.11 & -76.79 & -61.97 & -57.57 & -40.11 & -38.86 & -62.33 & -61.59 \\
\hline & & 4 & -77.87 & -80.61 & -57.87 & -78.98 & -39.05 & -47.28 & -61.26 & -61.47 \\
\hline & \multirow{3}{*}{$\left(2: 1 ; 45^{\circ}\right)$} & 2 & -71.50 & -70.35 & -56.21 & -54.65 & -46.12 & -45.43 & -54.39 & -55.43 \\
\hline & & 3 & -64.27 & -63.21 & -50.49 & -48.99 & -41.70 & -41.20 & -51.06 & -57.77 \\
\hline & & 4 & -60.54 & -59.34 & -47.53 & -45.93 & -39.85 & -39.10 & -49.76 & -48.00 \\
\hline & \multirow{3}{*}{$\left(2: 1 ; 90^{\circ}\right)$} & 2 & -50.37 & -53.38 & -55.88 & -55.85 & -62.13 & -52.47 & -50.30 & -72.17 \\
\hline & & 3 & -0.77 & -40.55 & -41.37 & -54.89 & $\begin{array}{l}-64.08 \\
\end{array}$ & -54.44 & -76.41 & -70.31 \\
\hline & & 4 & -7.30 & -57.26 & -40.70 & -58.50 & -52.73 & -53.71 & -70.65 & -72.77 \\
\hline \multirow{12}{*}{$\begin{array}{l}\text { Parent- } \\
\text { Vessel } \\
\text { Contact- } \\
\text { Angle }\end{array}$} & \multirow{3}{*}{$\left(3: 2 ; 45^{\circ}\right)$} & 2 & -30.02 & -39.28 & 8.79 & -6.97 & 50.42 & 65.33 & 50.62 & 45.68 \\
\hline & & 3 & -14.61 & -9.75 & 18.76 & 34.49 & 52.39 & 68.77 & 53.04 & 69.60 \\
\hline & & 4 & -9.91 & 2.53 & 22.84 & 168.94 & 53.01 & 64.97 & 54.07 & 172.95 \\
\hline & \multirow{3}{*}{$\left(3: 2 ; 90^{\circ}\right)$} & 2 & -47.82 & -75.55 & 5.44 & 19.69 & 52.65 & 24.49 & 30.00 & -1.13 \\
\hline & & 3 & -23.23 & 8.86 & 22.14 & 34.33 & 56.90 & 49.92 & 35.32 & 28.82 \\
\hline & & 4 & -0.20 & -14.36 & 29.47 & 25.03 & 56.61 & 25.55 & 36.83 & 17.84 \\
\hline & \multirow{3}{*}{$\left(2: 1 ; 45^{\circ}\right)$} & 2 & -79.66 & -83.12 & -22.78 & -26.85 & 35.42 & 26.49 & -23.01 & 24.80 \\
\hline & & 3 & -78.39 & -71.66 & -21.91 & -6.62 & 61.29 & 45.88 & 84.71 & 15.90 \\
\hline & & 4 & -68.07 & -47.59 & -12.22 & 11.62 & 29.08 & 46.83 & 57.80 & 48.92 \\
\hline & \multirow{3}{*}{$\left(2: 1 ; 90^{\circ}\right)$} & 2 & -64.58 & -73.46 & -22.20 & -28.79 & -4.82 & 10.18 & -16.09 & -22.06 \\
\hline & & 3 & -39.97 & -54.20 & -7.54 & -17.43 & -0.63 & 13.03 & -10.97 & -18.53 \\
\hline & & 4 & -24.99 & -44.91 & -0.80 & -14.33 & 1.44 & 11.62 & -7.82 & -22.00 \\
\hline
\end{tabular}

